\title{
¿Es la medicalización un fenómeno negativo? Un análisis de las consecuencias que suelen atribuirse a la medicalización
}

\author{
Is Medicalization a Negative Phenomenon? An Analysis of the \\ Consequences that are often Ascribed to Medicalization
}

\begin{abstract}
Resumen: En este artículo expondré un análisis de la valoración negativa de las consecuencias que suelen atribuirse al fenómeno de la medicalización y, partiendo de aquí, mi objetivo básico será mostrar que la medicalización no es en sí mismo un fenómeno negativo. Sólo lo será cuando se obtenga un valor negativo del cálculo entre, por un lado, la efectividad de la propuesta médica para solucionar el problema y, por otro lado, el balance entre los beneficios de dicha propuesta médica y sus posibles efectos secundarios; o bien, cuando se dé un contexto en el que el individuo, ya sea por desinformación o por una restricción externa (paternalismo médico), no tiene la libertad suficiente de escoger entre una propuesta médica y una propuesta no-médica.

Palabras clave: ética médica, medicalización, consecuencias negativas.
\end{abstract}

\begin{abstract}
ALBERTO OYA*
Abstract: In this paper I will analyze the negative character of those consequences that are often ascribed to the phenomenon of medicalization. Whit this, I will be arguing that medicalization is not a negative phenomenon per se. There are only two occasions in which medicalization will be a negative phenomenon. Firstly, when there is a negative result coming from the calculation between, on the one hand, the effectiveness of the medical way to solve the problem and, on the other hand, the balance between the benefits of the medical way and its possible negative side effects. Secondly, when the individual, either by disinformation or by an external restriction (medical paternalism), is not at liberty to choose between a medical way or a non-medical way.
\end{abstract}

Key words: medical ethics, medicalization, negative consequences.

\section{Introducción}

Entendemos por 'medicalización de la vida' o, simplemente, 'medicalización', el proceso por el cual aspectos, problemas o situaciones humanas que antes no eran considerados como pertenecientes al ámbito médico pasan a ser considerados como propios de la medicina. En otras palabras: se trata de definir bajo términos médicos una situación que hasta el momento había sido ajena a la medicina, al tiempo que se ofrece una nueva manera médica de afrontar dicha situación.

Fecha de recepción: 26/06/2015. Fecha de aceptación: 18/12/2015.

* Graduado en Filosofía (Universitat de Girona, UdG), Graduado en Geografía e Historia (Universidad Nacional de Educación a Distancia, UNED), y Máster en Filosofía Analítica (Universitat de Barcelona, UB). Actualmente cursando estudios de Doctorado en la Universitat de Girona. Contacto: alberto.oya.marquez@gmail.com 
Algunos ejemplos de procesos de medicalización acaecidos en las últimas décadas son los siguientes: la menopausia, con la introducción de maneras médicas de afrontar las situaciones propias de dicho período vital (me refiero especialmente a la llamada 'Terapia Hormonal Sustitutiva'); la impotencia sexual masculina, cuyo proceso de medicalización ha vivido un destacado avance tras la comercialización del citrato de sildenafilo ${ }^{1}$ a finales de los años noventa; el parto, pues mientras que hasta hace relativamente pocas décadas lo más común era alumbrar en casa y sin ningún tipo de asistencia médica, actualmente la regla general en los países desarrollados es precisamente la contraria, siendo realmente excepcionales los casos en los que se da a luz sin asistencia médica; el llamado 'síndrome post-vacacional', con el cual se redefinen los problemas que el individuo puede llegar a padecer al volver al trabajo tras treinta días de vacaciones y que pueden llegar a ser afrontados a partir de medidas médicas -por ejemplo: complejos vitamínicos; las relaciones sexuales, principalmente mediante el uso de métodos anticonceptivos médicos, empleados con la finalidad de prevenir los embarazos no deseados.

Asimismo, es necesario aclarar que aunque el propio proceso de medicalización comporta la redefinición de una situación humana bajo términos propios de la medicina, así como la aparición de una manera médica de afrontar esta situación recién redefinida, ello no quiere decir que estas situaciones sólo sean vistas como conflictivas tras su redefinición bajo términos médicos. Es decir: al menos por regla general, ${ }^{2}$ el fenómeno de la medicalización no se caracteriza por crear nuevos problemas sino por redefinir los ya existentes bajo términos médicos y ofrecer una solución de carácter médico para los mismos. Así, recuperando los ejemplos del párrafo anterior, puede decirse que la impotencia sexual masculina, algunos procesos vinculados a la etapa vital de la menopausia, la vuelta al trabajo tras los treinta días de vacaciones, el parto y los embarazos no deseados, ya antes del proceso de medicalización eran vistos, por una u otra razón, como situaciones conflictivas.

\section{Análisis de las consecuencias de la medicalización}

A continuación expondré las principales consecuencias que suelen atribuirse al fenómeno de la medicalización. Llama la atención que todas ellas, sin excepción alguna, son negativas. Mi objetivo será ofrecer un análisis crítico de la valoración negativa de dichas consecuencias.

Para mi análisis, es necesario trazar una distinción clara entre tres dimensiones o elementos de los procesos de medicalización: el proceso de medicalización en sí; lo que he denominado el cálculo entre la efectividad y los efectos secundarios de la propuesta médica; y lo que llamaré medicalización lucrativa. Por un lado, trataré la cuestión de si los procesos de medicalización son más beneficiales que perjudiciales, a través de lo que llamaré el cálculo entre la efectividad y los efectos secundarios de la propuesta médica. En segundo lugar, discutiré si la medicalización es, en sí mismo, y al margen de sus consecuencias, un fenómeno negativo. Por último, analizaré de forma específica los aspectos problemáticos propios de la llamada medicalización lucrativa. A pesar de que éste no suele ser el caso entre

1 Más conocido por su nombre comercial: Viagra.

2 Con la excepción de aquellos casos regidos por el patrón que he denominado medicalización lucrativa. Este concepto será desarrollado en el segundo apartado del presente artículo. 
los estudios que han tratado la cuestión de la medicalización, creo que estos tres conceptos deben distinguirse claramente. De hecho, la idea directriz del presente artículo es que no tener en cuenta esta necesaria distinción es una de las principales causas de la valoración negativa del fenómeno de la medicalización.

Con cálculo entre la efectividad y los efectos secundarios de la propuesta médica me refiero a la efectividad de la propuesta médica de afrontar el problema o situación que dicha propuesta pretende solucionar, ${ }^{3}$ junto con el balance entre sus posibles efectos secundarios negativos y sus beneficios. ${ }^{4}$ El cálculo entre la efectividad y los efectos secundarios es positivo en caso que la propuesta ponga fin al problema o sea una manera realmente útil para el individuo de tratar con él y no presente unos efectos secundarios negativos que pudieran ser más perjudiciales que el problema al cual se enfrenta; en caso contrario es negativo. ${ }^{5}$

Bajo la expresión de cálculo entre la efectividad y los efectos secundarios de la propuesta médica también puede englobarse el nivel de calidad del diagnóstico y pronóstico médicos. A pesar de que no será objeto de análisis en este artículo, vale la pena apuntar que de este modo la distinción también puede explicar aquellos casos que suelen usarse para justificar una valoración negativa del proceso de medicalización pero que, en realidad, tienen su origen en un diagnóstico médico de calidad insuficiente. Es decir: aquellos casos en que el problema no reside en que se quiera medicalizar una situación humana -esto es: dar una respuesta de tipo médico-, sino que el problema está en que se ofrece una solución médica a un individuo que en realidad no experimenta el problema que dicha solución pretende tratar -esto es: se produce un mal diagnóstico. Me refiero, por ejemplo, a aquellos jóvenes cuya hormona del crecimiento funciona a la perfección -lo único que sucede es que son de baja estatura- pero que, aún así, son diagnosticados por su médico con un trastorno hormonal del crecimiento, con la consiguiente prescripción de un tratamiento ineficaz - pues sólo es útil para aquellos que sí presentan un trastorno hormonal del crecimiento- y, por tanto, innecesario. ${ }^{6}$ Así pues, la calidad del diagnóstico y pronóstico médicos juegan un papel destacado en determinar la efectividad de la propuesta médica. Esto, sin embargo, no implica que la calidad del diagnóstico y pronóstico médicos jueguen ningún papel importante en el proceso de medicalización en sí -esto es: en la redefinición bajo términos médicos de situaciones antes ajenas al mundo de la medicina. Por ello, la calidad del diagnóstico y pronóstico médicos,

3 Así, por ejemplo, diré que la propuesta médica de tomar ciertos complejos vitamínicos para lidiar con el llamado 'síndrome post-vacacional' es efectiva si elimina u ofrece una buena manera de afrontar esta situación que el individuo, por una u otra razón, ve problemática. En caso contrario, diré que la propuesta carece de efectividad.

4 Diré que el balance es positivo cuando la propuesta médica no acarree unos efectos secundarios que pudieran ser más perjudiciales que la solución al problema al cual se enfrenta. De lo contrario, diré que el balance es negativo.

5 Aquí quizás se podría objetar que los procesos de medicalización están asociados a procesos de invención de enfermedades, y éstos a estrategias mercadotécnicas de creación de necesidades. Con lo cual se podría objetar que el problema o situación que la propuesta médica pretende solucionar no es realmente un problema. Sin embargo, el fallo de este argumento es, a mi parecer, que adolece de una visión excesivamente paternalista del ser humano; pues asume que los individuos son, por sí mismos, incapaces de identificar cuáles son sus verdaderos problemas.

6 Para más información acerca de este caso concreto véase: Conrad, P.; Leiter, V. «Medicalization, Markets and Consumers». Journal of Health and Social Behavior, vol. 45, número extra (2004), 158-176. 
aún siendo factores que determinan la efectividad de la propuesta médica, deben distinguirse del proceso de medicalización en sí.

Es también importante hacer notar que, aun cuando pueda acarrear ciertos efectos secundarios negativos, una práctica médica puede presentar un cálculo positivo siempre y cuando el balance entre sus beneficios y sus posibles efectos secundarios adversos sea positivo. Aunque en algunos casos pueda alcanzarse un consenso más o menos objetivo sobre esta cuestión, parece claro que en muchos otros responde a una decisión personal, subjetiva, siendo el propio individuo quien debe decidir por sí mismo si los beneficios de aplicar una determinada práctica médica compensan los posibles efectos adversos que ésta pueda conllevar. ${ }^{7}$

Con el término medicalización lucrativa me refiero a la promoción consciente promovida por un colectivo de medicalizar un problema o situación concreta con el único fin de obtener beneficios económicos, no teniéndose en cuenta la efectividad de la propuesta médica ofrecida para lidiar con tal problema o situación y siendo ésta un mero pretexto para lucrarse. ${ }^{8}$ En otras palabras: se trata de un proceso de medicalización en el que no se tiene en cuenta el cálculo entre la efectividad y los efectos secundarios de la propuesta médica. Por otro lado, cabe decir que aquellos colectivos que promueven un proceso de medicalización lucrativa no lo hacen de manera pública o explícita, sino que, como veremos más adelante, llevan a cabo un conjunto de acciones y estrategias para, por así decirlo, enmascarar sus verdaderas intenciones.

Una vez hechas estas distinciones es posible dividir las principales consecuencias que suelen atribuirse al proceso de medicalización en tres grupos. En primer lugar, tenemos aquellas que aun siendo propias de la medicalización sólo son negativas bajo la suposición de que lo que he denominado cálculo entre la efectividad y los efectos secundarios de la propuesta médica presenta un valor negativo. En el segundo grupo se encuentran aquellas consecuencias de la medicalización cuya valoración negativa es independiente de un resultado negativo del cálculo entre la efectividad y los efectos secundarios de la propuesta médica. Por último, tenemos aquellas consecuencias que reciben una valoración negativa por el hecho de consistir en el tipo de medicalización a la que he dado el nombre de medicalización lucrativa.

7 Quizás sea útil aquí un ejemplo. Piénsese en el consumo de andrógenos anabólicos: a pesar de que la mayoría de nosotros no afirmaríamos que su único efecto positivo - facilitar la hipertrofia muscular- compensa la posible presencia de sus numerosos efectos secundarios negativos - disfunciones hepáticas, alopecia, atrofia testicular, cambios de humor, aumento de la irritabilidad y la agresividad, etcétera-, parece claro que al menos para aquellos que los usan este único efecto positivo sí compensa sus numerosos efectos secundarios negativos. Por otro lado, imagínese ahora que existe un tratamiento médico que es efectivo en la cura del virus de la inmunodeficiencia humana (VIH) y cuyo efecto secundario adverso más destacado es la de generar tos seca mientras dura el tratamiento; en este caso, parece claro que todos nosotros estaríamos de acuerdo en que los beneficios de este tratamiento - esto es: la remisión de los síntomas más negativos provocados por el VIH, como es la aparición de enfermedades oportunistas- compensan la presencia de sus efectos secundarios adversos - esto es: la tos seca mientras durara el tratamiento.

8 Cuando hablo de 'colectivo' me refiero principalmente a la industria farmacéutica, al ser quien desarrolla y obtiene el mayor beneficio en la venta de un tratamiento médico, aunque también pueden incluirse aquí a aquellos profesionales sanitarios que puedan beneficiarse de este proceso, principalmente de manera económica -por ejemplo, mediante incentivos económicos por cada intervención o gratificaciones económicas por cada prescripción de un tratamiento. 


\subsection{Consecuencias de la medicalización cuya valoración negativa depende de un resultado} negativo del cálculo entre la efectividad y los efectos secundarios de la propuesta médica

La consecuencia más obvia del proceso de medicalización es el aumento del consumo de fármacos y/o de tratamientos e intervenciones médicas (Orueta Sánchez et al., 2011b, p.212). Esto, sin embargo, sólo se convierte en un hecho negativo si estas intervenciones médicas generan en los individuos efectos secundarios desagradables o, en todo caso, si el individuo cree que las ventajas proporcionadas por estas actuaciones médicas no compensan la presencia de dichos efectos secundarios -es decir: si el cálculo entre la efectividad y los efectos secundarios de la propuesta médica es negativo.

Otra de las consecuencias que suelen atribuirse a la expansión de la medicalización es la pérdida de las maneras tradicionales de afrontar nuestros problemas (Márquez y Meneu, 2007, p.81). En mi opinión, considerar esto como una consecuencia negativa tiene su origen en la confusión entre, por un lado, la cuestión del cálculo entre la efectividad y los efectos secundarios de la propuesta médica y, por otro, el proceso de medicalización propiamente dicho. Si el cálculo entre la efectividad y los efectos secundarios de la propuesta médica presenta un valor positivo -esto es: la forma médica de solucionar el problema, ya sea un tratamiento o una intervención, es realmente efectiva y el balance entre beneficios y efectos secundarios adversos es positivo-, no hay ningún motivo para otorgar mayor valor a la forma tradicional, no-médica, de solucionar un problema, cualquiera que sea éste, que la forma médica de solucionar el mismo problema. Es más: parece claro que en muchos casos la solución médica tiene mayor valor que la no-médica, en el sentido de que la primera soluciona de una manera más efectiva el problema al que se afronta. Un ejemplo de ello es la prevención del embarazo no deseado: si valoramos la pérdida de las formas tradicionales de afrontar un problema cualquiera como un fenómeno negativo entonces debería aceptarse, por ejemplo, que es algo negativo que se estén perdiendo las formas no-médicas de prevenir un embarazo no deseado -a saber: la práctica del coitus interruptus- en detrimento de las nuevas formas médicas -a saber: el uso de métodos anticonceptivos médicos. Con todo, resulta difícil encontrar alguna razón, más allá de las que conciernen al cálculo entre la efectividad y los efectos secundarios de la propuesta médica respecto al uso de estos métodos -esto es: su eficacia en la prevención de embarazos y el balance entre sus beneficios y sus posibles efectos secundarios negativos-, y puede dudarse de que la haya, para preferir la práctica del coitus interruptus al uso de métodos anticonceptivos médicos.

Si nos fijamos en las formas de tratar con determinadas situaciones relacionadas con el aspecto físico de los individuos es posible encontrar ejemplos muy similares al anterior, aunque mucho más llamativos. De entre ellos, me referiré a los que creo son los casos más interesantes: la calvicie y el descontento respecto el tamaño de los senos. Por el momento, parece que las únicas razones que pueden aducirse para preferir las formas no-médicas de afrontar la calvicie - por ejemplo: ponerse una peluca- o el descontento respecto el tamaño de los senos -por ejemplo: usar un sujetador que haga aparentar un tamaño de los senos diferente al natural-frente a las formas médicas de afrontar las mismas situaciones -tomar un determinado complejo vitamínico o llevar a cabo un injerto capilar, en el primer caso; modificar quirúrgicamente el tamaño de los senos, en el segundo- se basan en la ineficacia 
de estas últimas soluciones y/o en sus efectos secundarios desagradables -es decir: se basan en un resultado negativo del cálculo entre la efectividad y los efectos secundarios de la propuesta médica-, pero no justifican que de por sí sea mejor optar por una solución no-médica que una médica. Y esto último es precisamente lo que se necesita si se quiere justificar que la pérdida de las formas no-médicas de tratar con los problemas es una consecuencia negativa del proceso de medicalización.

$\mathrm{Al}$ basarse estos dos últimos ejemplos en situaciones que conciernen al aspecto físico de los individuos, podría responderse que todos y cada uno de nosotros debe aceptar su aspecto físico en su forma genuina. En otras palabras, que deben aceptarse los distintos aspectos de la vida humana -en este caso, los de carácter físico- tal y como aparecen de manera natural. De esta forma se pone fin a la cuestión, pues se afirma que el individuo no debe optar por ninguna acción para afrontar estas situaciones -es decir: ni recurriendo a las maneras tradicionales ni a las que proporciona la medicina-, pues en realidad no constituyen situaciones problemáticas. Aun dejando de lado el hecho de que proponer tal cosa adolece de una actitud ciertamente paternalista -pues impone al individuo el deber de aceptar algo que quizás no desee aceptar-, esta respuesta puede parecer a primera vista algo relativamente fácil de asumir; no obstante, trae consigo algunas consecuencias que son mucho más difíciles de aceptar por la mayoría de la población. Pues también implicaría, por ejemplo, el deber de aceptar las relaciones sexuales en su forma genuina; es decir: sin usar ningún tipo de método anticonceptivo -ni médico ni no-médico-y, en consecuencia, el deber de aceptar todos los embarazos no deseados que puedan resultar de una relación sexual cualquiera. Y es que una vez habiendo postulado este deber de aceptar las características físicas en su forma genuina, parece que no hay manera alguna de limitar este deber a las características físicas y no incluir en él también otros aspectos de la vida humana.

La profusión de políticas de prevención es otra de las consecuencias que suelen atribuirse al proceso de medicalización (Márquez y Meneu, 2007, p.72). Aunque las instituciones político-sanitarias son los principales responsables de esta práctica, cabe destacar también el importante papel de la industria farmacéutica. Mediante este tipo de políticas la industria farmacéutica amplía su cuota de mercado, abarcando no sólo a los que sufren un problema concreto o se ven obligados a afrontar una determinada situación que creen conflictiva, sino también a aquellos que no encontrándose en tal situación desean evitar verse en un futuro envueltos en ella.

Esto, sin embargo, no significa que las políticas de prevención tengan un móvil exclusivamente económico y respondan al patrón de lo que he denominado medicalización lucrativa. Tampoco significa que sean en ellas mismas un fenómeno negativo. La profusión de políticas de prevención sólo es un fenómeno negativo cuando el cálculo entre la efectividad y los efectos secundarios de la propuesta médica presenta un valor negativo; es decir: cuando no es realmente una prevención y/o cuando el balance entre sus beneficios y sus efectos secundarios adversos es negativo para el propio sujeto.

Para ejemplificar esto, haré mención a uno de los casos que suelen ofrecer los críticos de la medicalización para respaldar su valoración negativa acerca de la profusión de políticas de prevención: la relativamente reciente vacuna del virus del papiloma humano. Una buena parte de las críticas a esta vacuna se basan en su ineficacia para lograr el objetivo que pretende y/o los efectos secundarios negativos que produce, pero no en lo que propiamente 
la define como medida preventiva -esto es: el objetivo de prevenir el contagio del virus del papiloma humano. ${ }^{9}$ Sin embargo, la insuficiencia técnica de ciertas políticas preventivas no convierte la prevención en un fenómeno negativo. Cabe decir, además, que el consenso casi universal acerca del uso del preservativo como práctica de prevención frente al contagio de enfermedades de transmisión sexual -con la excepción de aquellos que aducen razones ajenas al ámbito de la medicalización; por ejemplo de carácter religioso- parece indicarnos que la idea de efectuar políticas de prevención, siempre y cuando sean eficaces y/o no produzcan efectos secundarios negativos,$^{10}$ es comúnmente valorada como un fenómeno positivo.

También suele decirse que el fenómeno de la medicalización va parejo a la transformación de factores de riesgo en enfermedades, con la consiguiente búsqueda y/o propuesta de una manera médica de tratar con ellos (Orueta Sánchez et al., 2011a, p.153). El ejemplo clásico es la osteoporosis. Una vez más, esto sólo se torna un fenómeno negativo cuando el resultado del cálculo entre la efectividad y los efectos secundarios de la propuesta médica presenta un valor negativo; es decir: cuando aquello que se conceptualiza como «factor de riesgo» no lo es realmente y/o cuando la propuesta médica no es eficaz en reducir dicho riesgo y/o cuando trae consigo más efectos secundarios negativos que beneficios.

Algo muy similar puede decirse respecto a otra de las consecuencias de la medicalización que suele valorarse como negativa, la medicalización de determinadas etapas de la vida. El ejemplo más comentado en la literatura especializada es la medicalización de la menopausia. Pues bien, mi respuesta es sencilla: la medicalización de la menopausia, o de cualquier otra etapa vital, es útil sólo si ofrece una manera deseable de afrontar las situaciones propias de dicho período vital. Y al contrario, en el caso de que las maneras médicas de tratar con dichas etapas vitales sean ineficaces y/o el individuo crea que las ventajas proporcionadas por estas actuaciones médicas no compensan la presencia de sus posibles efectos secundarios, entonces estaremos delante de un proceso de medicalización inútil, innecesario y no deseable.

\subsection{Consecuencias de la medicalización cuya valoración negativa es independiente de un resultado negativo del cálculo entre la efectividad y los efectos secundarios de la propuesta médica}

Creo que una de las consecuencias más serias que suelen aducirse en contra de la medicalización es que ésta trae consigo la concepción del individuo como objeto más que como sujeto (Parens, 2013, p.2). En otras palabras: la competencia del individuo de actuar y solucionar por sí mismo sus propios problemas se ve reducida, pues el individuo recurre a la medicina para afrontar sus problemas. Creo que esto es, además, la manera más seria y aparentemente más eficaz de argumentar a favor de una manera no-médica de afrontar los problemas respecto a una de carácter médico aún presuponiendo que el cálculo entre la efectividad y los efectos secundarios de la propuesta médica presenta un valor positivo.

9 Véase, por ejemplo: Forcades, T. [Publicación en línea] «Una reflexión y una propuesta en relación a la vacuna del VPH». 20 de noviembre de 2012. <http://teresaforcades.files.wordpress.com/2013/01/una-reflexic3b3i-una-proposta-en-relacic3b3-a-la-vacuna-del-virus-del-papilc2b7loma-humc3a0-cast1.pdf $>$ [Consulta: $10 / 03 / 2014]$.

$10 \mathrm{O}$, usando la terminología propuesta en este artículo: siempre y cuando el cálculo entre la efectividad y los efectos secundarios de la propuesta médica presente un resultado positivo. 
En mi opinión, esta pérdida o reducción de la competencia individual parece ser, hasta el momento, la principal crítica al fenómeno de la medicalización.

Como puede observarse, la fuerza de esta argumentación depende del supuesto que cuando un individuo recurre a la medicina adopta un papel meramente pasivo a la hora de solucionar sus problemas. No obstante, esto es falso -o al menos debería serlo-, pues si se garantiza que es el propio individuo quien libremente decide optar por una solución médica en detrimento de otra de carácter no-médico, puede concluirse que el individuo sigue manteniendo un papel activo a la hora de solucionar sus problemas o afrontar las situaciones que le parecen conflictivas; es decir: aunque el individuo recurra a la medicina, sigue siendo él mismo quien soluciona sus problemas, si bien es cierto que lo hace de manera mediata. Algo similar sucede en el modo en que la mayoría de nosotros afrontamos el problema, realmente cotidiano, de garantizar nuestra alimentación: lo hacemos de manera mediata -yendo a un supermercado- y no de manera inmediata -cultivando y cazando nuestros propios alimentos-; sin embargo, no consideramos la mediatez de nuestra solución como algo negativo pues, de hecho, suele preferirse comprar en el supermercado que aprender el arte de la horticultura.

He dicho que para que el individuo mantenga un papel realmente activo a la hora de solucionar sus problemas aún recurriendo a la medicina debe garantizarse que éste goce de la libertad suficiente para escoger entre las maneras médicas y las maneras no-médicas de afrontar las situaciones que se le presenten. La pregunta que debe responderse ahora es cómo garantizar tal cosa. Pues bien, para ello debe darse un contexto en el cual el individuo esté plenamente informado acerca de la efectividad de la propuesta médica y de sus posibles efectos secundarios, y que sea en él en quien recaiga la decisión última de optar o no optar por una solución de carácter médico. Esta situación se da cuando el individuo goza del acceso libre a información completa, real, verdadera e imparcial acerca de la efectividad de las maneras médicas de afrontar una determinada situación y cuando, rechazando el llamado paternalismo médico, el personal sanitario comprende que una de sus funciones principales es la de informar sobre la efectividad de la propuesta médica y de sus posibles efectos secundarios, pero que es en el propio paciente en quién debe recaer la decisión última. ${ }^{11}$

Otra consecuencia realmente importante de la medicalización es la reducción de los recursos disponibles para invertir en salud -como consecuencia del aumento de las intervenciones y/o tratamientos médicos-, con lo que éstos no pueden ser destinados a tratamientos y/o intervenciones que pudieran ser más necesarios, al tiempo que facilita la saturación del sistema sanitario público (Morell Sixto et al., 2009, p.494; Orueta Sánchez et al., 2011b, p.212). Es obvio que el proceso de medicalización comporta un incremento de las intervenciones y/o tratamientos médicos, y que éste, por el coste que conlleva, lleva aparejado una reducción de los recursos disponibles. Tan sólo quisiera apuntar que se trata de una cuestión puramente de gestión de los recursos disponibles y que afecta fundamentalmente al sector público, pues, aunque tanto en el sector público como en el privado los recursos son limitados, en el primer caso parece claro que la prescripción de tratamientos y/o intervenciones

11 Esta segunda condición es una realidad desde el momento en que se acepta el llamado principio de autonomía del paciente; esto es: que el individuo tiene el derecho de rechazar el tratamiento médico que le ha sido propuesto por el personal sanitario -independientemente de cuál sea éste-, así como de elegir aquel tratamiento que considere más adecuado para él mismo. 
médicos debe regirse con cierta mesura -podría, por ejemplo, establecerse un criterio según la necesidad de la intervención; esto es: garantizar primero los tratamientos que sean más necesarios y efectivos para mantener la integridad física del individuo- mientras que en el segundo caso no es necesaria una actitud tan mesurada en la administración de los recursos disponibles -en tanto que es el propio paciente quien asume la totalidad de los costes de su tratamiento. En cualquier caso, cabe decir que esta solución podría llegar a generar otro problema no menos importante que el de la saturación del sistema sanitario público; a saber: el incremento de la desigualdad. Y es que existiría la posibilidad de que en algunos casos solo unos pocos, los más favorecidos económicamente, pudieran costearse el precio de ciertos tratamientos médicos mientras que el resto no podría hacerlo, aumentándose así la desigualdad socioeconómica preexistente. Así pues, el fenómeno de la medicalización podría generar un problema de justicia distributiva. Sin embargo, esto por sí solo no justifica una valoración negativa del fenómeno de la medicalización: que los recursos sean limitados no implica que debamos dejar de hacer uso de ellos, lo que debemos hacer es encontrar una manera justa de distribuirlos.

La sanitarización de la sociedad y una mayor dependencia por parte de la población hacia la medicina es otro de los fenómenos parejos al proceso de medicalización (Márquez y Meneu, 2007, p.81; Orueta Sánchez et al., 2011b, p.212). Si bien esto es cierto, puede contestarse que desde el siglo XX toda la población occidental es sumamente dependiente de la electricidad y, no obstante, no parece que nadie pretenda rechazar la electrificación de la sociedad al tiempo que asume la consecuencia lógica de dicho rechazo, vivir su día a día sin electricidad.

Se dice también que la medicalización produce cierta obsesión en la población por lograr una salud perfecta y, al contrario, miedo y angustia frente a la enfermedad (Orueta Sánchez et al., 2011a, p.151). Por lo que refiere a esta consecuencia, no niego que el proceso de medicalización pueda producir cierta obsesión en la población por su estado de salud. Ahora bien, el problema está en que, para evitar esta obsesión, tendríamos que eliminar la posibilidad de ofrecer soluciones médicas, posiblemente eficaces, a problemas que afectan a la población; con lo cual, además de caer en una actitud paternalista, ${ }^{12}$ tendríamos, quizás, una población aparentemente menos angustiada, pero también una población más incapaz de dar respuesta a aquellas situaciones que cree conflictivas. Puede darse la misma respuesta a otra de las consecuencias que se derivan del proceso de la medicalización; a saber: el clasificar como enfermos a personas que antes de la medicalización podríamos considerar sanas (Cerecedo Pérez et al., 2013, p.435; Márquez y Meneu, 2007, p.81).

\subsection{Consecuencias negativas propias de la medicalización lucrativa}

La consecuencia principal de lo que he denominado medicalización lucrativa es la existencia de tratamientos y/o intervenciones médicas sin ningún tipo de interés en la efectividad que éstos tienen para solucionar el problema o situación a la que pretenden hacer frente. Esto hace que la gran mayoría de tratamientos y/o intervenciones médicos surgidos a raíz

12 Pues, por decirlo de manera directa, se prohíbe a la población una alternativa posible de afrontar sus problemas con el mero pretexto de que "no se obsesionen demasiado". 
de un proceso de medicalización lucrativa no tengan la más mínima efectividad e, incluso, que puedan resultar nocivos para la salud del individuo. Es innegable, por tanto, que la medicalización lucrativa es un fenómeno negativo y que debe ser evitado.

Es importante distinguir entre, por un lado, aquellos tratamientos y/o intervenciones médicas que forman parte de lo que he denominado medicalización lucrativa y, por otro lado, aquellos tratamientos y/o intervenciones médicas que reportan ingresos económicos. El punto a destacar es que el hecho de que un tratamiento médico reporte beneficios económicos no lo convierte automáticamente en un caso de medicalización lucrativa; solo será un caso de medicalización lucrativa si se deja a un lado la cuestión por lo que he llamado el cálculo entre la efectividad y los efectos secundarios de la propuesta médica y si su única y exclusiva motivación es la de obtener beneficios económicos.

Por otro lado, parece claro que no hay ningún proceso de medicalización que, pública y abiertamente, se justifique por el interés exclusivo de lucrar a quien la promueve. Los colectivos que promueven estos procesos de medicalización lucrativa suelen enmascarar su verdadero interés lucrativo haciéndolo pasar por un interés científico. A pesar de que este tema no será objeto de análisis en este artículo, cabe decir que una de las estrategias más utilizadas para este fin es la compra de expertos y de comités aparentemente científicos por parte de los colectivos que promueven estos procesos de medicalización lucrativa (Molina, 2013). De esta manera, estos colectivos consiguen dotarse de una aparente credibilidad científica con la que enmascarar sus verdaderos intereses lucrativos.

Además de intentar conseguir esta aparente credibilidad científica, para llevar a buen puerto un proceso de medicalización lucrativa se lleva a cabo un conjunto de acciones y estrategias por parte del colectivo que pretende lucrarse del mismo. Entre estas estrategias pueden destacarse las siguientes: tomar un síntoma común que puede significar cualquier cosa y hacerlo aparecer como el signo de una enfermedad seria; exagerar conscientemente los beneficios de un tratamiento médico, al tiempo que se obvian las desventajas y posibles efectos secundarios; manipular las estadísticas, estableciendo que hay un número de individuos que puede requerir de la solución médica mucho mayor que del que realmente hay (Vara, 2008, p.130; Moynihan et al., 2002, p.886).

\section{Conclusión: algunos procesos de medicalización constituyen un fenómeno positivo}

Llegados a este punto deberíamos preguntarnos si el fenómeno de la medicalización trae consigo alguna consecuencia positiva. Creo que la respuesta es afirmativa, siempre y cuando se dé un contexto en el que, por un lado, el individuo esté lo suficiente informado y goce de la libertad necesaria para poder escoger él mismo entre una manera médica y una manera no-médica de afrontar sus problemas y, por otro lado, que lo que he venido llamando a lo largo de este artículo como cálculo entre la efectividad y los efectos secundarios de la propuesta médica presente un valor positivo. Una vez que esto ocurre, el proceso de medicalización pone a disposición de la población una nueva y eficaz manera - de carácter médico- de solucionar sus problemas, por lo que éstos tienen una alternativa más, y una mayor variedad de opciones es siempre algo positivo. La presencia o inexistencia de estas condiciones parece constituir, además, un criterio efectivo para diferenciar entre procesos 
de medicalización que podríamos valorar de positivos y procesos de medicalización que podríamos calificar de negativos.

Además, es posible que el fenómeno de la medicalización produzca un impulso a las investigaciones en medicina y, con él, un avance en la efectividad de las propuestas médicas y una reducción de sus posibles efectos secundarios negativos. ${ }^{13}$ Por lo que es probable que la población tenga a su disposición un número cada vez mayor de propuestas médicas adecuadas para lidiar con sus problemas; y esto, siempre y cuando se den las condiciones previamente señaladas, es algo positivo. En cualquier caso, y para evitar malentendidos, cabe decir que con esto no estoy suponiendo que la medicina sea una ciencia ilimitada que pueda dar respuesta a todas y cada una de las situaciones humanas que podríamos considerar conflictivas, ni tampoco que tenga siempre una efectividad total; sólo estoy diciendo que la medicina parece no estar aún agotada y que parece tener la posibilidad de seguir desarrollándose.

\section{Bibliografía}

Cerecedo Pérez, MJ.; Tovar Bobo, M.; Rozadilla Arias, A. «Medicalización de la vida. Etiquetas de enfermedad: todo un negocio». Revista de Atención Primaria, vol. 45, 8 (2013), 434-438. Versión electrónica disponible en: <http://zl.elsevier.es/es/revista/ atencion-primaria-27/articulo/medicalizacion-vida-etiquetas-enfermedadtodo-90247085>

Conrad, P.; Leiter, V. «Medicalization, Markets and Consumers». Journal of Health and Social Behavior, vol. 45, número extra (2004), 158-176. Versión electrónica disponible en: <http://www.udel.edu/soc/tammya/socDev/conrad\%20leiter\%20on\%20 medicalization, $\% 20$ markets\%20and\%20consumers.pdf>

Conrad, P. «The Shifting Engines of Medicalization». Journal of Health and Social Behavior, vol. 46, 1, (2005), pp. 3-14. Versión electrónica disponible en: <http://psychopathology.fiu.edu/Articles/Conrad_05.pdf>

Forcades, T. [Publicación en línea] «Una reflexión y una propuesta en relación a la vacuna del VPH». 20 de noviembre de 2012. <http://teresaforcades.files. wordpress.com/2013/01/una-reflexic3b3-i-una-proposta-en-relacic3b3-a-la-vacuna-del -virus-del-papilc2b7loma-humc3a0-cast1.pdf>

Márquez, S.; Meneu, R. «La medicalización de la vida y sus protagonistas». Eikasia. Revista de Filosofia, vol. II, 8 (2007), 65-86. Versión electrónica disponible en: <http://www. revistadefilosofia.org/4Lamedicalizacion.pdf>

Molina Pérez, A. «¿Normal o patológico? El enfermo imaginario en tierra de nadie». Arbor, vol. 189, número 763 (2013). Versión electrónica disponible en: <http://arbor.revistas. csic.es/index.php/arbor/article/viewArticle/1869/2018>

Morell Sixto, ME.; Martínez González, C.; Quintana Gómez, JL. «Disease mongering, el lucrativo negocio de la promoción de enfermedades». Revista de Pediatría de Atención

13 Incentivado por el crecimiento de la demanda de tratamientos y/o intervenciones médicas que trae consigo el propio fenómeno de la medicalización. 
Primaria, vol. XI, 43 (2009), 491-512. Versión electrónica disponible en: <http://www. pap.es/files/1116-930-pdf/1062.pdf>

Moynihan, R.; Heath, I.; Henry, D. «Selling sickness: the pharmaceutical industry and disease mongering». British Medical Journal, vol. 321 (2002), 886-890. Versión electrónica disponible en: <http://www.ncbi.nlm.nih.gov/pmc/articles/PMC1122833/>

Orueta Sánchez, R.; et al. «Medicalización de la vida (I)». Revista Clínica de Medicina de Familia, vol. 4, 2 (2011a), 150-161. Versión electrónica disponible en: <http://scielo. isciii.es/scielo.php?script=sci_arttext\&pid=S1699-695X2011000200011>

Orueta Sánchez, R.; et al. «Medicalización de la vida (II)». Revista Clínica de Medicina de Familia, vol. 4, 3 (2011b), 211-218. Versión electrónica disponible en: <http://scielo. isciii.es/scielo.php?pid=S1699-695X2011000300005\&script=sci_arttext $>$

Parens, E. «On good and bad forms of medicalization». Bioethics, vol. 27, 1 (2013). Versión electrónica disponible en: <http://www.thehastingscenter.org/uploadedFiles/ About/People/Staff/Good\%20and\%20bad\%20forms \%20of\%20medicalization $\% 20$ early. pdf>

Vara, AM. «Cómo medicalizar la vida diaria: la creación de enfermedades o "disease mongering"». Evidencia -actualización en la Práctica Ambulatoria, vol. 11, 5 (2008), 130-132. Versión electrónica disponible en: <http://www.foroaps.org/files/ ygjytgkgyt.pdf> 